\title{
Nestin expression in repopulating mesangial cells promotes their proliferation
}

\author{
Christoph Daniel, Heinz Albrecht, Andrea Lüdke and Christian Hugo
}

We investigated whether the intermediate filament protein and neural stem cell marker nestin characterizes the glomerular progenitor/reserve cell population immigrating the glomerulus after mesangial cell (MC) injury in the rat (antiThy 1 nephritis). Nestin expression was investigated by immunohistochemistry and real-time PCR during anti-Thy 1 nephritis. Migration and proliferation assays were used to characterize the function of nestin in isolated MCs after nestin knockdown by siRNA. After MC injury during anti-Thy 1 nephritis, glomerular nestin was transiently increased during the repopulation phase. At the peak of mesangial proliferation and expansion (day 5) most OX-7-positive MCs expressed nestin largely colocalizing with the activation marker $\alpha$-smooth muscle actin and the proliferation marker PCNA. In contrast to a healthy, non-injured mesangium in vivo, MCs in culture are considered to be in an 'activated, injured state' and express nestin in a generalized distribution with condensed localization around the nucleus as well as intensive staining of cell protrusions such as filopodia. During cell cycle, the percentage of MCs with high nestin levels was increased during S- and G2-phase. Blocking of nestin using specific siRNA resulted in inhibition of cell proliferation but not cell migration. In conclusion, nestin is constitutively expressed in podocytes, but is a marker for repopulating MCs after experimental MC injury in vivo. Nestin promotes MC proliferation in vitro, suggesting a supporting role for nestin during repair reaction.

Laboratory Investigation (2008) 88, 387-397; doi:10.1038/labinvest.2008.5; published online 11 February 2008

KEYWORDS: stem cells; nestin; repopulation; proliferation; podocytes

Nestin is a class VI intermediate filament protein originally described as a marker for neuroepithelial stem cells. ${ }^{1}$ Later on, nestin was also described in non-neural progenitor and stem cells, such as the pancreatic islet ${ }^{2-5}$ as well as hematopoietic ${ }^{6}$ or hair follicle sheath progenitor populations, ${ }^{7,8}$ suggesting that its presence in cells may indicate multi-potentiality and regenerative potential. $^{9}$

Nestin is also expressed during developmental processes such as myogenesis of skeletal muscle and heart or the developing tooth bud, ${ }^{10}$ or the developing eye. ${ }^{11}$ Upon differentiation, nestin is downregulated, ${ }^{12,13}$ but reappears transiently after injury to muscle, ${ }^{14}$ brain ${ }^{15,16}$ and liver. ${ }^{17}$ In the kidney, nestin is also expressed during development, ${ }^{18,19}$ but its expression persists in the podocytes of mature glomeruli. $^{18,20}$ After podocytic injury, nestin is found to be upregulated within podocytes. ${ }^{20}$
In the anti-Thyl nephritis model, the intraglomerular mesangium is destroyed by an antibody-mediated reaction followed by repair via migrating and proliferating mesangial 'reserve' cells. ${ }^{21}$ More than $90 \%$ of these repopulating cells originate from the extraglomerular or hilar mesangium, whereas less than $10 \%$ of the repopulating cells may be derived from the bone marrow, ${ }^{21,22}$ supporting the concept of a predominantly locally regulated repair process similar to experimental ischemic tubulointerstitial injury of the kidney. ${ }^{23}$ Nevertheless, detailed characterization of these repopulating glomerular cells as well as markers to identify renal stem cells/progenitor cells are still lacking. ${ }^{24}$ Repair of the injured glomerulus requires both cell migration and cell proliferation.

This study was undertaken to characterize the expression of the stem cell marker nestin during mesangial cell (MC)

Department of Nephrology and Hypertension, University Erlangen-Nuremberg, Erlangen, Germany

Correspondence: Dr C Daniel, PhD, Department of Nephrology and Hypertension, University Erlangen-Nuremberg, Loschgestrasse 8, Erlangen 91054, Germany.

E-mail: Christoph.Daniel@uk-erlangen.de

Received 14 August 2007; revised 8 January 2008; accepted 9 January 2008 
repopulation and to investigate nestins role in $\mathrm{MC}$ migration and proliferation.

\section{MATERIALS AND METHODS Animal Model}

Mesangial proliferative glomerulonephritis (anti-Thy1 model)

The animal studies were performed in accordance with the Internal Animal Review Board (Regierung von Mittelfranken: 621-2531.31-04/03). Experimental mesangial proliferative glomerulonephritis was induced in six Sprague-Dawley rats (150-200 g; Charles River, Sulzfeld, Germany) by a single injection of $1 \mathrm{mg} / \mathrm{kg}$ of the mouse monoclonal anti-Thy 1 antibody OX-7 (purified from supernatant of OX-7 hybridoma cells (purchased from the European Collection of Animal Cell Cultures, Porton Down, Salisbury, UK)). Renal biopsies, as described previously, ${ }^{21}$ were performed on days 3 and 5, and the experiment was finished on day 8 . Additionally, biopsies of a group from six healthy controls were taken to investigate constitutive nestin expression in the kidney. To evaluate nestin mRNA during anti-Thyl nephritis, we performed a second independent experiment using four rats each for glomerular preparations from anti-Thy 1 nephritic kidneys $0,1,3,5$ and 8 days after induction of the disease. Glomerular preparations were done by the standard sieving procedure as described previously. ${ }^{25}$

\section{Biopsies from healthy human and mouse kidneys}

Normal human renal tissue $(n=6)$ was obtained from kidneys surgically excised because of the presence of a localized neoplasm. Tissues utilized for this study were obtained from macroscopically normal portions of kidney located at some distance from the neoplastic process. Kidneys used in this study were obtained from the Department of Pathology at the University Erlangen-Nuremberg (Erlangen, Germany). Kidney biopsies from healthy mice were obtained from six male C57bl6 mice with a body weight of 20-25 g.

\section{Immunohistochemistry of renal biopsies and cultured glomerular cells}

Renal biopsies from mice and rats were fixed in methyl Carnoy's solution and human biopsies in formalin. Tissues were embedded in paraffin and cut into 3- $\mu \mathrm{m}$ sections for indirect immunoperoxidase staining or immunofluorescence as described elsewhere. ${ }^{21,26}$

The following antibodies were used in this study: nestin, clone rat 401 a murine $\mathrm{IgG}_{1} \mathrm{mAb}$ specific for rat and murine nestin (Chemicon International, Temecula, CA, USA); nestin, clone $4 \mathrm{D} 4$ a murine IgG1 $\mathrm{mAB}$ specific for murine nestin (Biotrend, Köln, Germany); nestin, a murine $\operatorname{IgG}_{1} \mathrm{mAb}$ specific for human nestin (Chemicon); OX-7, a murine $\operatorname{IgG}_{1}$ mAb specific for rat CD90 expressed on MCs (Serotec Ltd, Oxford, UK); ${ }^{21} \alpha$-smooth muscle actin ( $\alpha$-sm-actin), a murine $\operatorname{IgG}_{2} \mathrm{mAb}$ specific for activated MCs (Dako, Hamburg, Germany); ${ }^{27}$ RECA-1, a murine IgG mAb specific for rat endothelial cells (Serotec Ltd); ${ }^{28,29}$ ezrin, a murine IgG1
$\mathrm{mAb}$ specific for ezrin (Labvision Corporation, Fremont, CA, USA); and ${ }^{26}$ proliferating-cell nuclear antigen (PCNA), a murine IgG1 mAb (clone PC10) specific for the PCNA (Dako, Glostrup, Denmark). Negative controls for immunostaining included either deleting the primary antibody or substitution of the primary antibody with equivalent concentrations of an irrelevant murine monoclonal antibody or preimmune rabbit/goat IgG.

To determine glomerular nestin in Thy-1 nephritic kidneys in each biopsy, 40 cortical glomerular cross-sections were evaluated by computerized morphometry (Visitron Systems $\mathrm{GmbH}$, Puchheim, Germany) in a blinded manner.

\section{Immunohistochemical double stainings}

To determine the number of nestin-positive proliferating cells in nephritic kidneys, double immunostaining for PCNA, a marker for cell proliferation, and nestin was performed as described previously. ${ }^{25}$ As secondary antibody, we used a rat adsorbed biotinylated horse anti-mouse IgG (Vector, Burlingame, USA), which was detected by horseradish peroxidase-conjugated avidin D (Vector). The peroxidase substrate 3,3'-diaminobenzidine (substrate kit; Vector) was used without nickel resulting in a brown staining (nestin) and Histogreen (Vector) was used for green nuclear staining (PCNA).

For colocalization studies, the anti-nestin antibody was directly labeled with the fluorochrome Alexa 488 using the Zenon mouse IgG labeling Kit (Molecular Probes Europe, Leiden, The Netherlands) following the manufacturer's instructions. Cell-specific markers (ezrin, Ox-7, RECA) or activation marker (sm-actin) were detected using a highly cross-absorbed donkey anti-mouse IgG antibody conjugated with Alexa 555 (Molecular Probes Europe).

\section{Real-time quantitative RT-PCR}

For evaluation of nestin mRNA levels during anti-Thyl nephritis, RNA was purified from isolated glomeruli collected 0 , $1,3,5$ and 8 days after nephritis induction. Glomeruli were lysed in RLT buffer and RNA was purified using Rneasy micro-columns (both from Qiagen, Hilden, Germany), with subsequent DNAse digestion following the manufacturer's instructions. Reverse transcription was performed using reverse transcription reagents from ABgene, Hamburg, Germany, following the manufacturer's instructions.

Real-time RT-PCR was performed on a TaqMan ${ }^{\circledR} \mathrm{ABI}$ 7000 Sequence detection system using the Mastermix (all ABgene). After an initial hold of $15 \mathrm{~min}$ at $95^{\circ} \mathrm{C}$, samples were cycled 40 times at $95^{\circ} \mathrm{C}$ for $15 \mathrm{~s}$ and $60^{\circ} \mathrm{C}$ for $60 \mathrm{~s}$. The cDNA content of each sample was compared with $18 \mathrm{~S}$ as housekeeping gene following the $\Delta \Delta C_{\mathrm{t}}$ technique. ${ }^{30}$ Both genes were quantified using the SYBR-GREEN method. For nestin mRNA detection, we used the Rn_Nes_1_SG QuantiTect Primer Assay (Qiagen) and for the housekeeping $18 \mathrm{~S}$ (forward primer 5'-TTGATTAAGTCCCTGCCCTTTGT; reverse primer 5'-CGATCCGAGGGCCTCACTA-3'). 


\section{Cell culture}

Mouse MCs were cultured from isolated glomeruli using standard sieving procedure ${ }^{31}$ and characterized by positive staining for $\alpha$-sm-actin and $\alpha 8$-integrin, and the lack of the epithelial marker WT-1, endothelial marker CD-31 and the macrophage/monocyte marker CD68. MCs were grown in DMEM containing $10 \%$ FCS, insulin $(5 \mu \mathrm{g} / \mathrm{ml})$ and penicillin/streptomycin at $37^{\circ} \mathrm{C}$ and $5 \% \mathrm{CO}_{2}$.

\section{Flow cytometry analysis}

Nestin expression was analyzed by flow cytometry using a mouse anti-nestin antibody (Chemicon). For investigation of nestin's role during $\mathrm{MC}$ proliferation, we simultaneously stained for DNA using propidium iodide and nestin. First, nestin expression was investigated in growing, non-synchronized MCs. Second, for time-course experiments and analysis of proliferation, MCs were starved for 2 days in serum-deprived culture medium, followed by stimulation with either $10 \%$ FCS or $20 \mathrm{ng} / \mathrm{ml}$ platelet-derived growth factor (PDGF) for $0,7,15$ and $24 \mathrm{~h}$. MCs were fixed over night in ice-cold $65 \%$ methanol after detatchment of growing cells from petri dishes by trypsin treatment. After washing with PAB buffer (PBS containing 2\% BSA and 0.01\% sodium azide), cells were permeabilized with $0.1 \%$ Triton $\mathrm{X}-100$ in PBS for 15 min at $37^{\circ} \mathrm{C}$, followed by washing with PAB buffer. For staining of DNA, RNA was digested by RNAse A $\left(1 \mathrm{mg} / \mathrm{ml}\right.$ in PBS) for $12 \mathrm{~min}$ at $37^{\circ} \mathrm{C}$, and for $40 \mathrm{~min}$ at room temperature. After washing with $\mathrm{PAB}$ buffer, cells were incubated with anti-nestin antibody (diluted 1:400 in PAB buffer for $2 \mathrm{~h}$ at $4^{\circ} \mathrm{C}$ ), washed again with PAB buffer, followed by incubation with an FITC-conjugated rabbit anti-mouse IgG antibody (Dako). After removal of non-bound antibody, MCs were facultatively stained with propidium iodide $(50 \mu \mathrm{g} /$ $\mathrm{ml}$ in PBS) and analyzed by flow cytometry analysis (EPICX XL; Beckman-Coulter, Krefeld, Germany) using EXPO32 ADC software (Beckman-Coulter). Cells were defined as nestin-negative when a similar FACS signal was detected than in controls without primary antibody. DNA staining by propidium iodide was also used for cell-cycle analysis. Percentages of G1, S and G2 cell-cycle phase were analyzed using Multicycle software (Phoenix Flow Systems, San Diego, USA).

\section{Transfection with nestin siRNA}

Mouse MCs were transfected with two HP GenomeWide siRNAs against nestin, a negative-control siRNA, or transfection reagent alone all purchased by Qiagen. Cells were transfected with 1, 10 and $50 \mathrm{nM}$ siRNA each using HiPerfect (Qiagen) as transfection reagent following the manufacturer's instructions. All tested nestin siRNA concentrations were able to downregulate nestin $48 \mathrm{~h}$ after transfection. Best specific effects without off-target effects were achieved using $10 \mathrm{nM}$ siRNA. Consequently, experiments using $10 \mathrm{nM}$ siRNA were shown. Downregulation of nestin was evaluated by flow cytometry analysis and cells were tested in proliferation and migration assays.

To exclude inhibition of cell-cycle proliferation due to toxicity of siRNA, we measured lactate dehydrogenase activity in the culture media of siRNA- vs non-treated MCs. Lactate dehydrogenase activity was analyzed using LDH cytotoxicity detection kit purchased from Takara Bio Inc. (Shiga, Japan) following the manufacturer's instructions. With the exception of nestin siRNA B, which induced some low cytotoxicity of about $10 \%$, all other investigated samples did not express any cytotoxicity.

\section{Proliferation assay}

Proliferation of MCs treated either with nestin siRNA or control siRNA was monitored by bromodeoxyuridine (BrdU) uptake during S-phase using a cell proliferation ELISA (colorimetric) purchased from Roche Diagnostics $\mathrm{GmbH}$, Mannheim, Germany, following the manufacturer's instructions. One thousand siRNA-transfected mouse MCs were seeded per well of a microtiter plate and starved for 2 days in culture medium supplemented with $0.1 \%$ FCS. Pulsing with BrdU was performed for $2 \mathrm{~h}$ a day after stimulation with $10 \%$ FCS. Proliferative cell activity of a representative experiment was shown. All assays were performed in sextuplicate and each experiment was performed a minimum of three times.

\section{In vitro cell migration assay}

MC migration was investigated using a Transwell 48-well modified Boyden chamber and $0.1 \%$ gelatine-coated polyvinyl-pyrrolidone-free polycarbonate membranes with $8-\mu \mathrm{m}$ pore size (Neuroprobes Inc., Gaithersburg, MD, USA). Two days after transfection with siRNAs, 28000 mouse MC per well were loaded into the upper chamber. The bottom wells were filled with $40 \mu \mathrm{g} / \mathrm{ml}$ fibronectin, used as the chemoattractant, or $0.1 \% \mathrm{BSA}$, used as a negative control. The Boyden chamber was incubated for $5 \mathrm{~h}$ at $37^{\circ} \mathrm{C}$ and $5 \% \mathrm{CO}_{2}$ to allow migration of cells through the membrane. Cells that had transmigrated were stained with Qick diff and three fields of vision per well were analyzed using phase-contrast microscopy at a $\times 250$ magnification. All assays were performed in triplicate and each experiment was performed a minimum of three times.

\section{Statistical analysis}

All values are expressed as mean \pm s.d. Statistical significance (defined as $P<0.05$ ) was evaluated with SPSS software (SPSS $\mathrm{GmbH}$, Munich, Germany) using the Student's $t$-test and ANOVA.

\section{RESULTS}

In Healthy Kidneys, Nestin is Expressed by Podocytes of Mouse, Rat and Human Origin

Nestin expression was detected in adult kidney biopsies of three different species, including mouse, rat and human 
origin, using immunohistochemistry with three different nestin-specific antibodies. Glomeruli from all three species show specific nestin staining restricted to podocytes (Figure $1 \mathrm{a}-\mathrm{c}$ ), whereas resting MCs are devoid of nestin. Since this study was undertaken to characterize the expression of nestin during MC repopulation, we evaluated nestin during anti-Thyl nephritis after severe MC injury.

\section{Nestin is Transiently Expressed De Novo by Repopulating MCs During Glomerular Repair after Mesangial Injury}

Few days after induction of anti-Thyl nephritis, glomeruli start to regenerate the destroyed mesangium via repopulation by a coordinated migratory and proliferative response from the hilar region. On day 3 after disease induction, besides predominant nestin staining in podocytes (Figure 1d, P), repopulating glomerular cells in the hilar region (Figure 1d and $\mathrm{h}$ ) are also positive for the intermediate filament protein nestin. De novo expression of nestin showing a typical mesangial pattern becomes more prominent during the mesangial proliferative phase of anti-Thyl nephritis on day 5, demonstrating a nearly twofold total glomerular upregulation compared with healthy control rats (Figure 1e and g). In addition, podocytic nestin expression also appears to be elevated. The significant upregulation of nestin persists up to day 8 in nephritic glomeruli, but was slightly decreased compared with day 5 values (Figure if and g). Glomerular nestin mRNA as assessed by quantitative PCR reaches its maximum on day 3 during anti-Thyl nephritis, with an upregulation of $2.31 \pm 0.77$ fold compared with levels found in healthy controls (Figure 1h). Five days after disease induction, nestin mRNA decreases to $1.4 \pm 0.2$ fold upregulation, decreasing further on day 8 to levels slightly lower than in healthy control glomeruli (Figure 1h).

To confirm mesangial expression of nestin during anti-Thyl nephritis, we performed double stainings using cell-specific markers (Figure 2). Nestin colocalizes with immigrating Ox-7-positive MCs on day 3 (Figure 2a-c, arrows) and day 5 (Figure $2 \mathrm{~d}-\mathrm{f}$ ), and with ezrin-positive podocytes (Figure $2 \mathrm{~g}-\mathrm{i}$ ) 5 days after induction of anti-Thy 1 nephritis. Since MCs are lacking nestin in the healthy kidney, further colocalization studies were performed to characterize the phenotype (activated, proliferative) of nestin-positive MCs. We found colocalization of nestin with $\alpha$-sm-actin, a cytoskeletal marker for activated MCs, in most but not all areas on day 5 of anti-Thyl nephritis (Figure 2j-1). In contrast, staining for the endothelial marker RECA-1 is clearly separated from nestin-positive cells (Figure $2 \mathrm{~m}-\mathrm{o}$ ) during anti-Thyl nephritis.

\section{Nestin is Expressed in MCs Under In Vitro Conditions}

Nestin positivity in cultured MCs is consistent with an indication of activation/phenotypic change, since cultured MCs in contrast to a healthy, non-injured mesangium in vivo, are considered to be in an 'activated, injured state' (Figure 3a and d). Cultured MCs show a generalized nestin distribution with condensed localization around the nucleus as well as marked staining of cell protrusions such as filopodia (Figure 3d). In actively spreading MCs, nestin was found within lamellipodia colocalizing with vinculin (Figure $3 \mathrm{a}-\mathrm{c}$ ). Nestin expression was increased in dividing cells (Figure $3 \mathrm{a}$ and e, d) compared with that in non-dividing cells (Figure $3 \mathrm{a}$ and e, nd), but remained at the same expression level within the filopodia.

\section{PDGF and FCS Induced Nestin Expression in MCs In Vitro}

In MCs growth-arrested by serum deprivation, we found 10-20\% cells to be negative for nestin (Figure $4 \mathrm{a}$ and $\mathrm{b}$ ), and less than 10\% highly positive for nestin (Figure 4a and b; Table 1). Nestin was significantly upregulated by both FCS and PDGF stimulation (Figure 4; Table 1). Stimulation with PDGF induced significantly higher nestin expression in MCs starting after $7 \mathrm{~h}$ and reaching its maximum with 3.5 -fold increased levels of highly nestin positive cells at $24 \mathrm{~h}$ (Figure 4a, c, e and g; Table 1). Similar induction of nestin expression was observed after stimulation with 10\% FCS, reaching significance $15 \mathrm{~h}$ after induction (Figure $4 \mathrm{~b}, \mathrm{~d}$ and f; Table 1). Both stimuli increased nestin expression before significant changes in cell cycle could be detected (Table 1). Statistically significant changes in the distribution of cell-cycle stages were found not before $24 \mathrm{~h}$ after the proliferative stimulus by FCS or PDGF (Table 1).

\section{Nestin is Differentially Expressed During Cell Cycle and Promotes Cell Proliferation}

Double staining of nestin during anti-Thyl nephritis with the proliferation marker PCNA revealed that nestin-positive MCs are highly proliferative. On day 3 after nephritis induction when the mesangial repopulation process starts,

Figure 1 Nestin in normal healthy glomeruli of different species and during anti-Thy1 nephritis. Detection of nestin by immunohistochemistry (brown color) shows typical podocytic staining pattern without mesangial positivity in healthy glomeruli from mouse (a), rat (b) and human (c) origin. Three days after induction of anti-Thy 1 nephritis, besides podocytes nestin positivity (brown color) is also seen in MCs (d, see MC) predominantly in close proximity to the hilar region where repopulation of the glomerulus starts (d, see $\mathbf{h}$ ). On day 5, nestin staining of the mesangium is peaking and mostly equally distributed within all glomerular areas (e). On day 8 of anti-Thy 1 disease, when the migratory and proliferative response of the mesangium is ceasing, mesangial expression of nestin is markedly decreasing (f) as quantified by computer-assisted morphometry (g). Glomerular nestin mRNA was investigated by real-time PCR using glomeruli isolated from healthy rats and 1, 3, 5 and 8 days after anti-Thy 1 nephritis induction. Nestin mRNA levels were normalized to $18 \mathrm{~S}$ mRNA and compared with healthy controls (h). 
as well as later on day 5 at the peak of repair, repopulating cells are predominantly nestin/PCNA double positive (Figure $5 \mathrm{a}$ and $\mathrm{b}$, arrows).
To determine whether de novo expression of nestin by MCs is involved in MC proliferation, we investigated nestin expression during cell cycle of MCs and cell
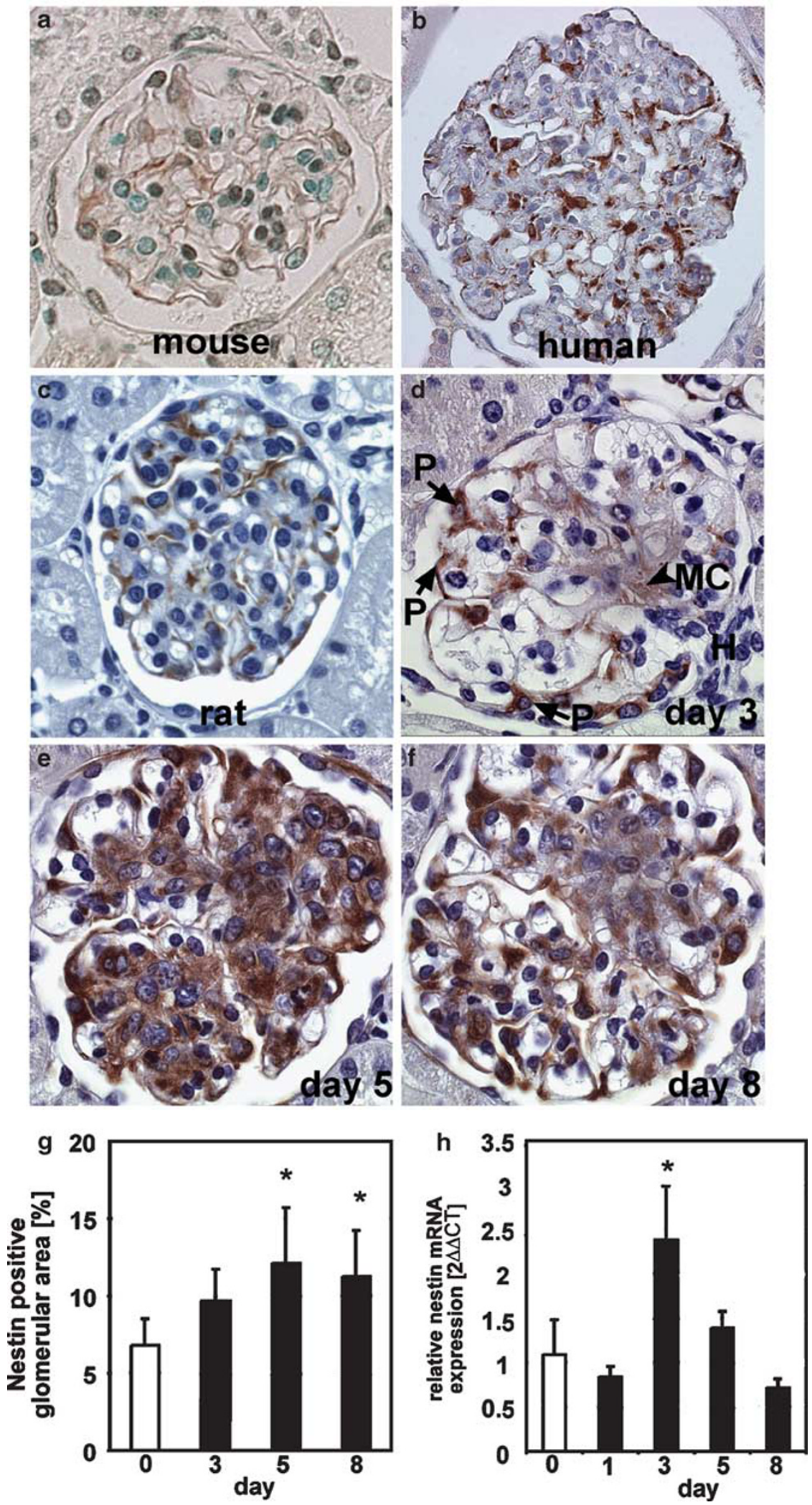


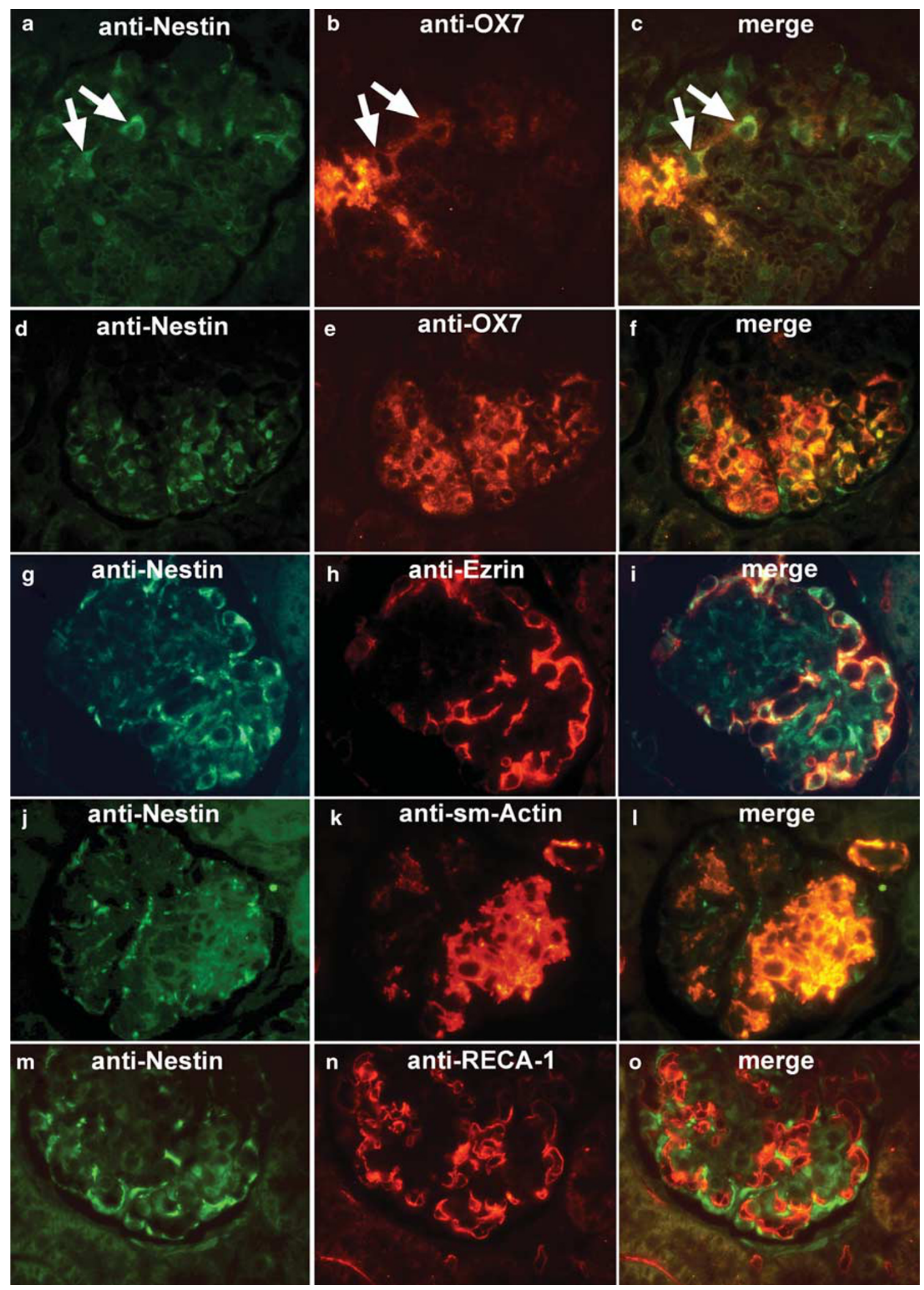

Figure 2 Colocalization studies confirm de novo expression of nestin by activated and proliferating MCs. Double immunostaining in kidney biopsies from anti-Thy 1 nephritic rats on day 5 confirms that nestin $(\mathbf{a}, \mathbf{d}, \mathbf{g}, \mathbf{j}, \mathbf{m}$, green fluorescence) colocalizes with the MC marker Ox-7 on days 3 (b, red fluorescence; $\mathbf{c}$, yellow fluorescence) and 5 (e, red fluorescence; $\mathbf{f}$, yellow fluorescence), the podocyte marker ezrin (h, red fluorescence; $\mathbf{i}$, yellow fluorescence) and with the activation marker for MCs, $\alpha$-sm-actin (k, red fluorescence; l, yellow fluorescence). In contrast, glomerular endothelial cells as identified by anti-RECA-1 antibody (n, red fluorescence) show no colocalization with nestin-positive cells ( $\mathbf{m}$, $\mathbf{o}$, green fluorescence). 

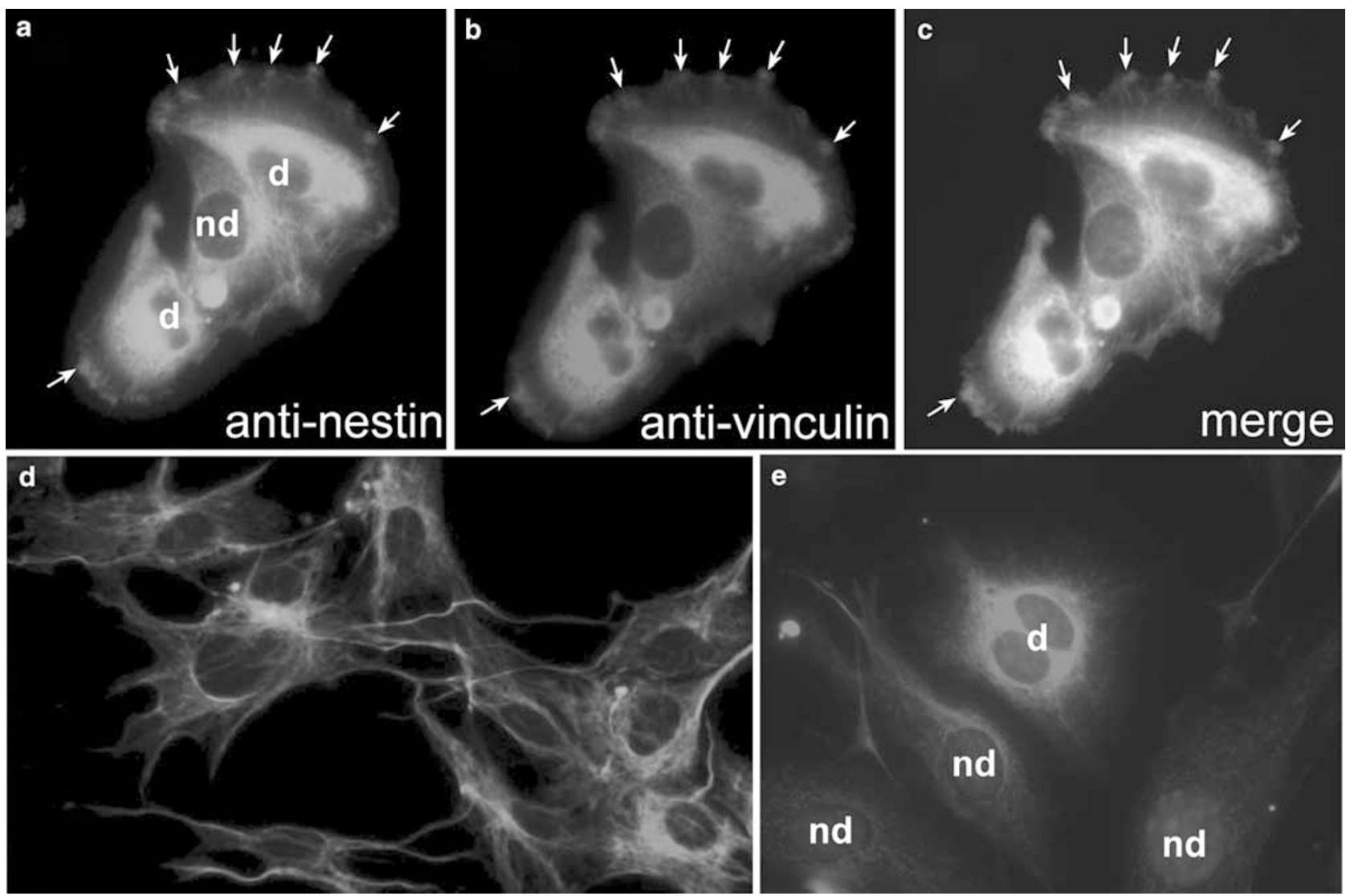

merge

Figure 3 Nestin is expressed in cultured MCs and localizes to cell extensions. Double immunofluorescence staining with nestin (a, green fluorescence), vinculin (b, red fluorescence) and nuclear staining (c, blue DAPI staining) using spreading MCs demonstrates nestin/vinculin colocalization in the cytoplasm and lamellipodia (c, arrows). In established MC cultures, cellular nestin is distributed within the cytoplasm preferentially around the nucleus, but also within cell extension such as filopodia (d, red fluorescence). Dividing MCs (a, e, d, green and red fluorescence) showed much higher nestin positivity around the nuclei than found in not dividing MCs (a, e, nd, green and red fluorescence).

proliferation after blocking of nestin expression by siRNA transfection.

In vitro experiments with double staining for DNA and nestin showed that nestin was differentially expressed during cell cycle of non-synchronized cultured MCs (Figure 5d). This differential nestin expression was similar in synchronized MCs treated with either transfection reagent only or a negative-control siRNA (Figure 5f). During S-phase, the percentage of MCs with high nestin levels was nearly doubled and was 2.5-fold higher during G2-phase compared with cells of the G1-phase (Figure 5d, black bars; Figure 5f, white bars). Two different siRNA sequences clearly downregulated nestin expression in MCs $48 \mathrm{~h}$ after transfection compared with cells treated with control siRNA (Figure 5e and $\mathrm{f}$ ). The extent of nestin inhibition by siRNA was similar during all cell cycle stages, producing five times less cells highly positive for nestin compared with negative controls (Figure 5f). The influence of nestin on cell proliferation was investigated by BrdU incubation as well as cell-cycle analysis. Nestin siRNA significantly and reproducibly inhibited MC proliferation by $10-18 \%$, depending on the effectivity of the siRNA block (Figure $5 \mathrm{e}$ and g). Consequently, the percentage of MCs in
G1-phase was significantly higher and in S-phase significantly lower (Figure 5h).

\section{Downregulation of Nestin did not Affect MC Migration}

Since MCs immigrating during repopulation are both proliferating and migrating, we also investigated the consequences of nestin blocking using a Transwell migration assay. Forty-eight hours after siRNA transfection, two nestin-specific siRNAs did not change MC migration compared with MCs treated with control siRNA or transfection reagent only (Figure 6).

\section{DISCUSSION}

Nestin is an intermediate filament protein and a marker for neuroepithelial and non-neural stem cells, ${ }^{1}$ suggesting that its presence in cells may indicate multi-potentiality and regenerative potential. ${ }^{9}$ In this study, we examined whether nestin could be a marker for repopulating mesangial precursor/reserve cells after injury in the anti-Thyl model in the rat.

The first major finding in this study is that nestin, besides its constitutive podocytic expression, is de novo expressed by 

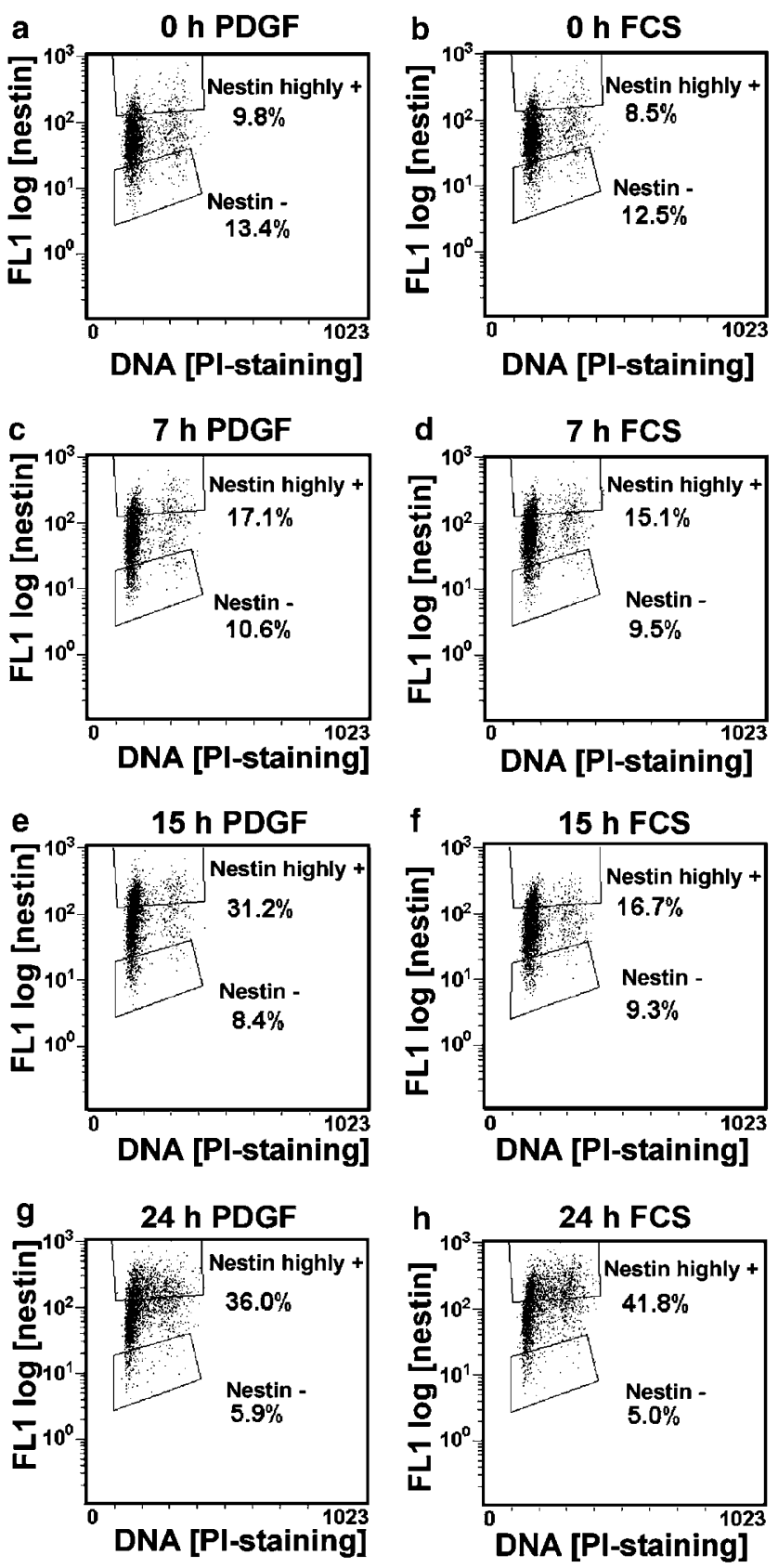

Figure 4 Nestin is upregulated by PDGF and FCS in MCs in vitro. Flow cytometry plots of representative experiments for nestin stimulation by PDGF and FCS are shown. Serum-deprived $\operatorname{MCs}(\mathbf{a}, \mathbf{b})$ were stimulated with PDGF for 0 (a), 7 (c), 15 (e) and $24 \mathrm{~h}(\mathbf{g})$, stained for nestin ( $y$-axis) and DNA by propidium iodide ( $x$-axis), and were analyzed by flow cytometry. Analogous experiments were performed using 10\% FCS stimulation for 0 (b), 7 (d), 15 (f) and 24h (h). Cells were analyzed for highly nestin positive cells (upper gate) and nestin-negative cells (lower gate).

repopulating MCs after injury. After severe mesangiolysis, most of the destroyed mesangium is replaced within days by migrating and proliferating Ox-7-positive MCs originating from the hilar and extraglomerular mesangium, restoring the glomerular architecture in a coordinated manner from the
Table 1 Nestin expression can be induced by PDGF and FCS

\begin{tabular}{lcccc}
\hline \multirow{2}{*}{$\begin{array}{l}\text { Experimental } \\
\text { group }\end{array}$} & $\begin{array}{l}\text { Cells highly } \\
\text { positive for } \\
\text { nestin (\%) }\end{array}$ & \multicolumn{3}{c}{ Distribution of cell-cycle stages } \\
\cline { 3 - 5 } & & $\mathrm{G} 1$ & $\mathrm{~S}$ & $\mathrm{G} 2$ \\
\hline 0 h PDGF & $8.9 \pm 0.9$ & $76.5 \pm 1.0$ & $13.1 \pm 1.5$ & $10.5 \pm 0.4$ \\
7h PDGF & $15.2 \pm 1.9^{*}$ & $76.6 \pm 7.4$ & $12.2 \pm 4.0$ & $11.3 \pm 3.4$ \\
15 h PDGF & $26.9 \pm 4.4^{*}$ & $79.8 \pm 4.2$ & $10.2 \pm 1.5$ & $10.0 \pm 2.7$ \\
24 h PDGF & $45.8 \pm 9.8^{*}$ & $44.1 \pm 3.5^{*}$ & $40.3 \pm 7.3^{*}$ & $15.6 \pm 3.8$ \\
0h FCS & $8.0 \pm 0.4$ & $78.9 \pm 3.5$ & $11.6 \pm 2.9$ & $9.6 \pm 0.6$ \\
7h FCS & $19.8 \pm 8.9$ & $78.5 \pm 7.1$ & $11.9 \pm 0.6$ & $9.7 \pm 1.6$ \\
15 h FCS & $28.3 \pm 9.9^{* *}$ & $76.4 \pm 4.2$ & $14.2 \pm 2.1$ & $9.5 \pm 2.1$ \\
24h FCS & $50.8 \pm 7.7^{* *}$ & $41.7 \pm 4.0^{* *}$ & $44.1 \pm 4.7^{* *}$ & $14.2 \pm 0.7^{* *}$
\end{tabular}

Serum-deprived mesangial cells were stimulated either by incubation with $20 \mathrm{ng} / \mathrm{ml} \mathrm{PDGF}$ or $10 \% \mathrm{FCS}$ for 7,15 or $24 \mathrm{~h}$. Cells were stained for nestin and DNA for analysis of nestin expression and cell cycle by flow cytometry. *Indicates significant changes of PDGF-stimulated cells, and ** indicates significant FCS-stimulated changes compared with non-stimulated controls.

hilus toward the glomerular periphery. ${ }^{21}$ Here, timely repair of the injured glomerular endothelium appears to be required for successful healing reaction of the glomerular architecture, ${ }^{32}$ and may indicate the importance of endothelialmesangial interaction for this repair process. While these immigrating glomerular cells express the typical mesangial marker protein Ox-7, most of them are also positive for $\alpha$-sm-actin, indicating a different or 'activated' phenotype compared with normal healthy MCs that are $\alpha$-sm-actin negative. ${ }^{27}$ Coexpression of nestin with the activation marker $\alpha$-sm-actin was recently reported in myofibroblasts after induction of obstructive nephropathy, ${ }^{33}$ suggesting a role of nestin in cells with an 'activated' phenotype. It is a matter of debate whether these immigrating cells are normal MCs acquiring a transient phenotype switch during repair, or whether they are primarily different from normal MCs and differentiate into MCs by loosing their specific phenotypical characteristics $(\alpha$-sm-actin positivity etc.) once they are incorporated in the glomerular architecture. While this study was not designed to answer the latter question, transient nestin positivity of these immigrating cells suggests involvement in cell migration and multi-potentiality. As reported for glia cells, ${ }^{34}$ we found that stimulation with PDGF also resulted in upregulation of nestin in cultured MCs. Since the major proproliferative cytokine PDGF is known to be upregulated in MCs during anti-Thyl nephritis, ${ }^{35}$ this is a potential mechanism for nestin induction in this mesangioproliferative model. We found coexpression of nestin with the proliferation marker PCNA in most glomerular areas during anti-Thyl nephritis, suggesting that nestin is potentially involved in cell proliferation.

The second major finding of this study indicates that nestin is not just upregulated in repopulating and 

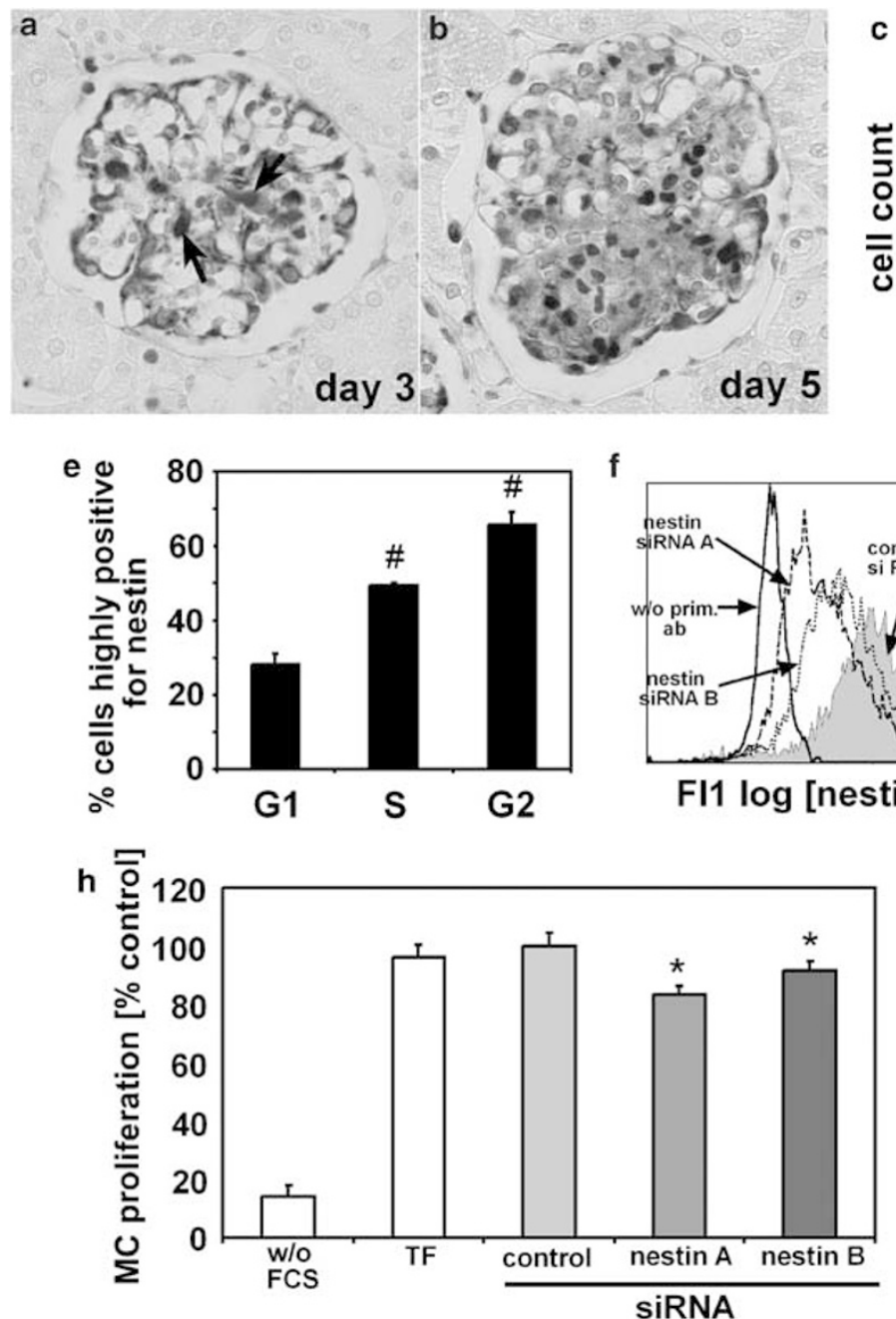

f

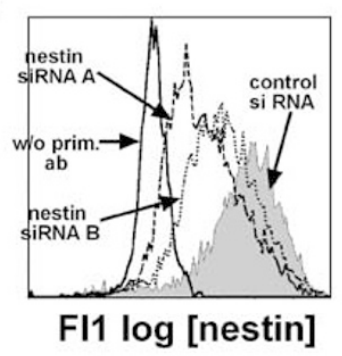

C

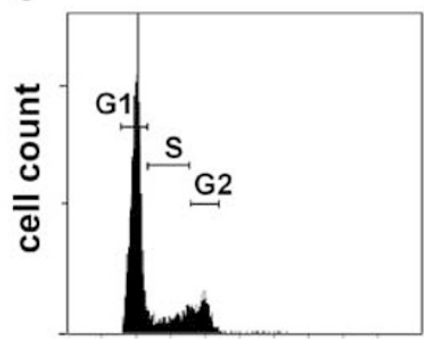

$\mathrm{F} 13 \operatorname{lin}$ [DNA]

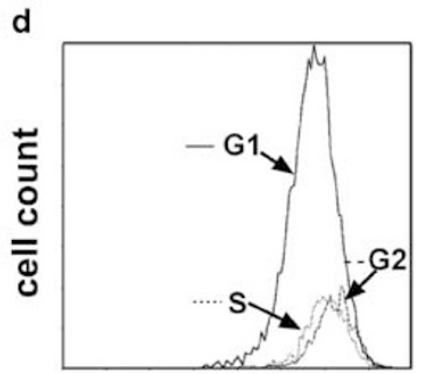

FI1 log [nestin]
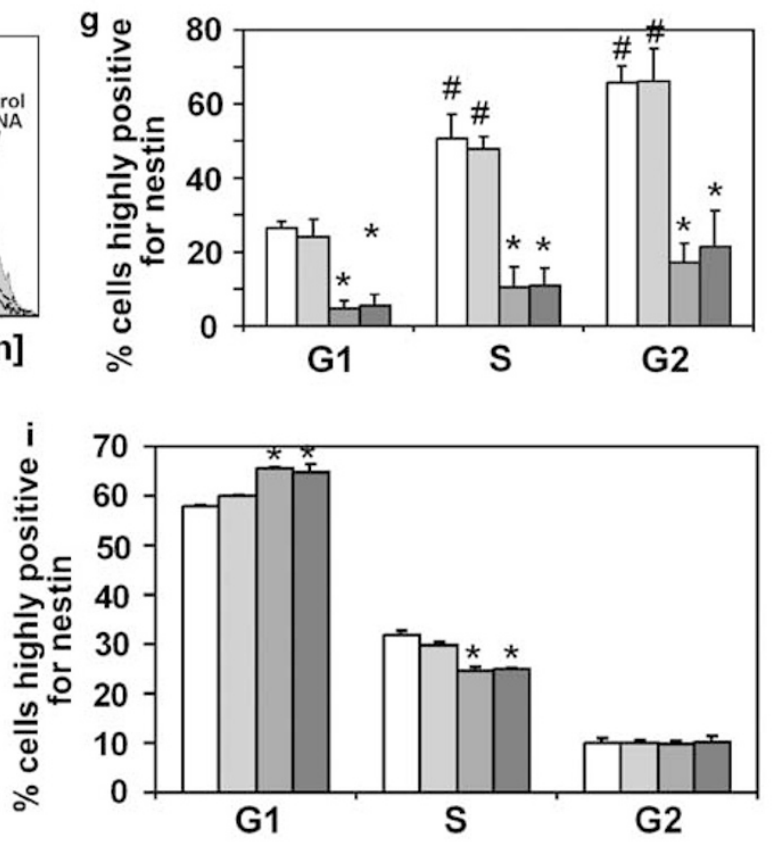

\begin{tabular}{|lll|}
\hline non-treated cells & $\square$ transfection reagent only & $\square$ nestin A siRNA \\
& $\square$ neg control siRNA & $\square$ nestin B siRNA
\end{tabular}

Figure 5 Nestin is differentially expressed during cell cycle and promotes cell proliferation. Double immunostaining in kidney biopsies from anti-Thy1 nephritic rats on days 3 and 5 for nestin (brown color) and the proliferation marker PCNA (green nuclei) demonstrates that most nestin-positive cells in a typical mesangial position are also proliferating (a, day 3, arrows; b, day 5). After staining of DNA in cultured MCs by propidium iodide, cell-cycle analysis was performed by flow cytometry analysis (c). In parallel, MCs were stained for nestin for cell-cycle phase-dependent analysis (d). The percentage of cells expressing high levels of nestin were determined during G1-, S- and G2-phases, and demonstrated increased nestin expression during S- and G2-phases (e). " Indicates statistic significant changes between G1- and S- phases $(P<0.008)$ and $\mathrm{G} 2$-phase $(n=4, P<0.0001)$ tested by two-way ANOVA analysis. Transfection of MCs with siRNA-targeting nestin successfully downregulated nestin expression as demonstrated by flow cytometry analysis (f, $\mathbf{g}, n=4 ; P<0.0001$ ). MC proliferation, as measured by BrdU incorporation (h) or cell-cycle analysis (i), was significantly reduced in MCs after transfection with nestin siRNA compared with MCs transfected with nonspecific control siRNA. * Indicates statistical significant changes between cells treated with transfection reagent only and siRNA-treated cells tested by two-way ANOVA analysis $(n=4 ; P<0.05)$.

proliferating MCs, but also has a function in MC proliferation but not in migration. Earlier studies described nestin as a marker of highly proliferative ${ }^{36-39}$ and migrating cells. ${ }^{37}$ In the kidney, a recent study showed nestin-positive cells migrating from the renal papilla to the cortex after ischemia/ reperfusion, ${ }^{40}$ indicating a potential role of nestin also for renal cell migration. We found that nestin is a marker that promotes MC proliferation but not migration in vitro. In this study, blocking of nestin resulted in mild but significant and consistent inhibition of MC proliferation, indicating that nestin promotes MC proliferation, but is not essential for this process. Since blocking of nestin in MCs by siRNA was not complete, the relevance of nestin on MC proliferation may even be more important than seen in our studies. The mechanisms and involvement of nestin in cell proliferation and migration are not completely understood. Nestin preferentially forms intermediate filaments by assembly with other intermediate filament proteins like vimentin and desmin, ${ }^{41}$ contributing to mechanical stability of cells. ${ }^{20}$ Vimentin and desmin are both found to be upregulated in 


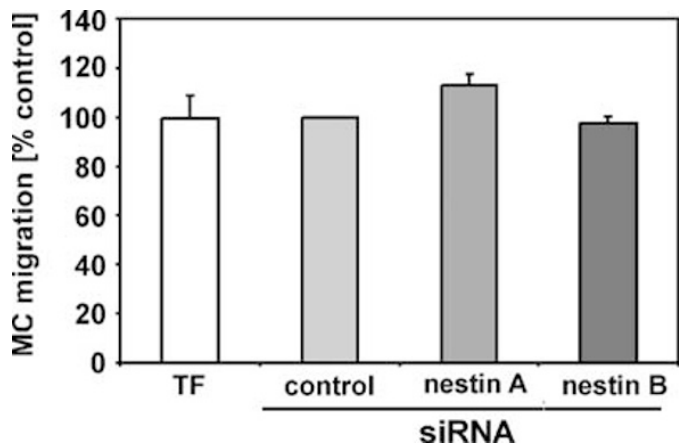

Figure 6 Nestin downregulation by siRNA did not affect MC migration. Transfection of MCs with two different siRNAs targeting nestin did not alter MC migration compared with a nonspecific control siRNA using a Boydenchamber migration assay using fibronectin as chemoattractant. The graph represents mean values with standard deviations from three similar experiments. The control of each experiment, using transfection reagent only (TF), was set to $100 \%$ migration. Efficacy of siRNA-targeting nestin is shown in Figure 5. Statistic significance was tested using one-way ANOVA analysis.

MCs during anti-Thyl nephritis. ${ }^{27}$ Nestin is furthermore suggested to act as a linker or cross-bridge protein between intermediate filaments, microfilaments and microtubules. ${ }^{42,43}$ In rapidly dividing and migrating cells assembly and disassembly of filaments need to be strictly regulated. In a neuronal stem cell line, nestin was found to be reorganized during mitosis in. ${ }^{44}$ Phosphorylation of nestin by different kinases leads to disassembly of nestin filaments during mitosis ${ }^{44}$ or development of neuronal precursor cells. ${ }^{45}$

This role of nestin during MC proliferation is further supported by the additional findings that nestin expression is clearly regulated during cell-cycle progression and that nestin knockdown by siRNA does affect cell-cycle progression in cultured MCs. In cultured MCs, nestin is markedly increased around nuclei of dividing cells and is strongly upregulated during S- and G2-phases compared with S1-phase. One potential mechanism of nestin-mediated cell-cycle progression is its role in regulation of vimentin. Disassembly of vimentin by nestin becomes further important, since disassembled, non-filamentous vimentin was able to move protein particles at high speeds along microtubles with molecular motors. ${ }^{10}$ Here, rapid transport of precursor molecules between different cytplasmatic compartments may allow nestin-positive cells to divide more rapidly. ${ }^{10}$ Differential expression of nestin during cell cycle suggests that nestin's function can be regulated by phosphorylation and upregulation of its expression. In other cell types, nestin's involvement was found in both cell proliferation and migration. In contrast to the results in this study, blocking of nestin by antisense expressing plasmids in human neuroblastoma cells resulted in reduction of cell motility by more than $40 \%$ in vitro, ${ }^{46}$ suggesting that involvement of nestin in cell migration is dependent on the cell type. Furthermore, blocking of nestin in cultured podocytes prevents the formation of foot processes, ${ }^{18}$ suggesting a role for nestin in organization of the cytoskeleton.

In conclusion, whereas podocytes demonstrate constitutive nestin expression in normal healthy glomeruli, diseased glomeruli specifically express nestin de novo in repopulating MCs. Our studies in vitro suggest that nestin is a marker for repopulating cells, which promotes MC proliferation but not migration.

\section{ACKNOWLEDGEMENTS}

This study was supported in part by a grant from Interdisciplinary center of clinical investigation (IZKF) project $A 12$ and the Deutsche

Forschungsgememeinschaft (SFB 423, TP B6). Technical help of Ulrike Goller, Andrea Braun, Susanne Weber, Birgit Hausknecht and Tanja Christ is gratefully acknowledged. We are grateful to the Department of Pathology at the University Erlangen-Nuremberg (Erlangen, Germany) for providing human renal tissue samples. Portions of this work were presented at the American Society of Nephrology annual meeting, San Diego, November 12-17, 2003 (J Am Soc Nephrol, vol. 14, November 2003).

1. Lendahl U, Zimmerman LB, McKay RD. CNS stem cells express a new class of intermediate filament protein. Cell 1990;60:585-595.

2. Hunziker E, Stein M. Nestin-expressing cells in the pancreatic islets of Langerhans. Biochem Biophys Res Commun 2000;271:116-119.

3. Zulewski $\mathrm{H}$, Abraham EJ, Gerlach MJ, et al. Multipotential nestinpositive stem cells isolated from adult pancreatic islets differentiate ex vivo into pancreatic endocrine, exocrine, and hepatic phenotypes. Diabetes 2001;50:521-533.

4. Lechner A, Leech CA, Abraham EJ, et al. Nestin-positive progenitor cells derived from adult human pancreatic islets of Langerhans contain side population (SP) cells defined by expression of the ABCG2 (BCRP1) ATPbinding cassette transporter. Biochem Biophys Res Commun 2002;293:670-674.

5. Abraham EJ, Kodama S, Lin JC, et al. Human pancreatic islet-derived progenitor cell engraftment in immunocompetent mice. Am J Pathol 2004; 164:817-830.

6. Walczak P, Chen N, Hudson JE, et al. Do hematopoietic cells exposed to a neurogenic environment mimic properties of endogenous neural precursors? J Neurosci Res 2004;76:244-254.

7. Li L, Mignone J, Yang M, et al. Nestin expression in hair follicle sheath progenitor cells. Proc Natl Acad Sci USA 2003;100:9958-9961.

8. Hoffman RM. The pluripotency of hair follicle stem cells. Cancer Biol Ther 2006;5:232-233.

9. Wiese C, Rolletschek A, Kania G, et al. Nestin expression-a property of multi-lineage progenitor cells? Cell Mol Life Sci 2004;61:2510-2522.

10. Michalczyk K, Ziman M. Nestin structure and predicted function in cellular cytoskeletal organisation. Histol Histopathol 2005;20:665-671.

11. Yang J, Bian W, Gao X, et al. Nestin expression during mouse eye and lens development. Mech Dev 2000;94:287-291.

12. Zimmerman L, Parr $B$, Lendahl $U$, et al. Independent regulatory elements in the nestin gene direct transgene expression to neural stem cells or muscle precursors. Neuron 1994;12:11-24.

13. Lothian C, LendahI U. An evolutionarily conserved region in the second intron of the human nestin gene directs gene expression to CNS progenitor cells and to early neural crest cells. Eur J Neurosci 1997:9:452-462.

14. Vaittinen $S$, Lukka R, Sahlgren $C$, et al. The expression of intermediate filament protein nestin as related to vimentin and desmin in regenerating skeletal muscle. J Neuropathol Exp Neurol 2001;60:588-597.

15. Krum JM, Rosenstein JM. Transient coexpression of nestin, GFAP, and vascular endothelial growth factor in mature reactive astroglia following neural grafting or brain wounds. Exp Neurol 1999;160:348-360.

16. Duggal N, Schmidt-Kastner R, Hakim AM. Nestin expression in reactive astrocytes following focal cerebral ischemia in rats. Brain Res 1997;768:1-9.

17. Gleiberman AS, Encinas JM, Mignone JL, et al. Expression of nestingreen fluorescent protein transgene marks oval cells in the adult liver. Dev Dyn 2005;234:413-421. 
18. Chen J, Boyle $S$, Zhao $M$, et al. Differential expression of the intermediate filament protein nestin during renal development and its localization in adult podocytes. J Am Soc Nephrol 2006;17: 1283-1291.

19. Wagner $\mathrm{N}$, Wagner $\mathrm{KD}$, Scholz $\mathrm{H}$, et al. The intermediate filament protein nestin is expressed in the developing kidney and heart and might be regulated by the Wilms' tumor suppressor Wt1. Am J Physiol Regul Integr Comp Physiol 2006;291:R779-R787.

20. Zou J, Yaoita E, Watanabe $Y$, et al. Upregulation of nestin, vimentin, and desmin in rat podocytes in response to injury. Virchows Arch 2006;448:485-492.

21. Hugo C, Shankland SJ, Bowen-Pope DF, et al. Extraglomerular origin of the mesangial cell after injury. A new role of the juxtaglomerular apparatus. J Clin Invest 1997;100:786-794.

22. Ito $T$, Suzuki A, Imai E, et al. Bone marrow is a reservoir of repopulating mesangial cells during glomerular remodeling. J Am Soc Nephrol 2001;12:2625-2635.

23. Lin F, Moran A, Igarashi P. Intrarenal cells, not bone marrow-derived cells, are the major source for regeneration in postischemic kidney. J Clin Invest 2005;115:1756-1764.

24. Lin F, Igarashi P. Searching for stem/progenitor cells in the adult mouse kidney. J Am Soc Nephrol 2003;14:3290-3292.

25. Hugo C, Pichler R, Meek R, et al. Thrombospondin 1 is expressed by proliferating mesangial cells and is up-regulated by PDGF and bFGF in vivo. Kidney Int 1995;48:1846-1856.

26. Hugo C, Nangaku M, Shankland SJ, et al. The plasma membrane-actin linking protein, ezrin, is a glomerular epithelial cell marker in glomerulogenesis, in the adult kidney and in glomerular injury. Kidney Int 1998;54:1934-1944.

27. Johnson RJ, lida $H$, Alpers $C E$, et al. Expression of smooth muscle cell phenotype by rat mesangial cells in immune complex nephritis. Alpha-smooth muscle actin is a marker of mesangial cell proliferation. J Clin Invest 1991;87:847-858.

28. Kang $\mathrm{DH}$, Kanellis J, Hugo $\mathrm{C}$, et al. Role of the microvascular endothelium in progressive renal disease. J Am Soc Nephrol 2002;13:806-816.

29. Iruela-Arispe L, Gordon K, Hugo C, et al. Participation of glomerular endothelial cells in the capillary repair of glomerulonephritis. Am J Pathol 1995;147:1715-1727.

30. Dimmler A, Haas CS, Cho S, et al. Laser capture microdissection and real-time PCR for analysis of glomerular endothelin-1 gene expression in mesangiolysis of rat anti-Thy 1.1 and murine Habu Snake Venom glomerulonephritis. Diagn Mol Pathol 2003;12:108-117.

31. Johnson RJ, Garcia RL, Pritzl P, et al. Platelets mediate glomerular cell proliferation in immune complex nephritis induced by anti-mesangial cell antibodies in the rat. Am J Pathol 1990;136:369-374.
32. Daniel C, Renders L, Amann K, et al. Mechanisms of everolimus-induced glomerulosclerosis after glomerular injury in the rat. Am J Transplant 2005:5:2849-2861.

33. Sakairi T, Hiromura $K$, Yamashita $S$, et al. Nestin expression in the kidney with an obstructed ureter. Kidney Int 2007;72:307-318.

34. Gallo V, Armstrong RC. Developmental and growth factor-induced regulation of nestin in oligodendrocyte lineage cells. J Neurosci 1995;15(1 Pt 1):394-406.

35. Ostendorf $T$, Kunter $U$, van Roeyen $C$, et al. The effects of plateletderived growth factor antagonism in experimental glomerulonephritis are independent of the transforming growth factor-beta system. J Am Soc Nephrol 2002;13:658-667.

36. Namiki J, Tator $\mathrm{CH}$. Cell proliferation and nestin expression in the ependyma of the adult rat spinal cord after injury. J Neuropathol Exp Neurol 1999;58:489-498.

37. Mothe AJ, Tator $\mathrm{CH}$. Proliferation, migration, and differentiation of endogenous ependymal region stem/progenitor cells following minimal spinal cord injury in the adult rat. Neuroscience 2005;131: 177-187.

38. Rice $A C$, Khaldi $A$, Harvey $H B$, et al. Proliferation and neuronal differentiation of mitotically active cells following traumatic brain injury. Exp Neurol 2003;183:406-417.

39. Chouaf-Lakhdar L, Fevre-Montange M, Brisson C, et al. Proliferative activity and nestin expression in periventricular cells of the adult rat brain. Neuroreport 2003;14:633-636.

40. Patschan D, Michurina T, Shi HK, et al. Normal distribution and medullary-to-cortical shift of nestin-expressing cells in acute renal ischemia. Kidney Int 2007;71:744-754.

41. Herrmann $\mathrm{H}$, Aebi $\mathrm{U}$. Intermediate filaments and their associates: multi-talented structural elements specifying cytoarchitecture and cytodynamics. Curr Opin Cell Biol 2000;12:79-90.

42. Hirokawa N, Glicksman MA, Willard MB. Organization of mammalian neurofilament polypeptides within the neuronal cytoskeleton. J Cell Biol 1984;98:1523-1536.

43. Hisanaga S, Hirokawa N. Structure of the peripheral domains of neurofilaments revealed by low angle rotary shadowing. J Mol Biol 1988;202:297-305.

44. Sahlgren CM, Mikhailov A, Hellman J, et al. Mitotic reorganization of the intermediate filament protein nestin involves phosphorylation by cdc2 kinase. J Biol Chem 2001;276:16456-16463.

45. Sahlgren $\mathrm{CM}$, Mikhailov $\mathrm{A}$, Vaittinen $\mathrm{S}$, et al. $\mathrm{Cdk5}$ regulates the organization of nestin and its association with p35. Mol Cell Biol 2003;23:5090-5106.

46. Thomas SK, Messam CA, Spengler BA, et al. Nestin is a potential mediator of malignancy in human neuroblastoma cells. J Biol Chem 2004:279:27994-27999. 